\title{
O gradiente rio-barragem do reservatório de Sobradinho afeta a composição florística, riqueza e formas biológicas das macrófitas aquáticas?
}

\author{
Are floristic composition, richness and life forms of aquatic macrophytes affected by the dam-river \\ gradient of the Sobradinho Reservoir?
}

Edson Gomes de Moura Júnior ${ }^{1,5}$, Maria Carolina de Abreu ${ }^{3}$, William Severi ${ }^{2}$

\& Giulliari Alan da Silva Tavares Lira ${ }^{4}$

\begin{abstract}
Resumo
O presente estudo teve por objetivo avaliar a riqueza, formas biológicas e composição florística de macrófitas aquáticas ao longo do eixo rio-barragem de um reservatório no Nordeste do Brasil. Foram estabelecidas seis estações de amostragem ao longo de suas regiões lótica, de transição e lêntica. As coletas ocorreram nos meses de junho e julho de 2008 (período de estiagem), e em janeiro e fevereiro de 2009 (período chuvoso). Foram coletados indivíduos em estagio fértil ou vegetativo, tendo as espécies sido categorizadas quanto às formas biológicas e os percentuais de frequência de ocorrência. A similaridade florística entre os pontos de amostragem foi calculada utilizando-se o índice de Jaccard. Foram identificadas 43 espécies, das quais 18 estiveram presentes na região lótica, 16 na de transição e 25 na lêntica. Salvinia auriculata Aubl. e Paspalum repens P.J. Bergius ocorreram em 100\% das unidades amostrais. As regiões lótica e de transição contemplaram, predominantemente, espécies anfíbias e emergentes, diferindo da região lêntica, onde espécies flutuantes, emergentes e submersas foram mais evidentes. A similaridade entre os ecossistemas lótico e de transição foi maior que $50 \%$, enquanto que entre esses ambientes e a região lêntica foi menor que $25 \%$. Palavras-chave: florística, hábito, reservatórios, similaridade.
\end{abstract}

\begin{abstract}
This study aimed to evaluate aquatic-macrophyte richness, life forms and floristic composition along the longitudinal axis of Sobradinho Reservoir - Bahia. Six sampling stations were selected along the lotic, transition and lentic stretches. Sampling was conducted in June and July 2008 (drought season), and in January and February 2009 (rainy season). Individuals in both fertile and vegetative stages were sampled and the species were classified according to life form and occurrence frequency. Floristic similarity among sampling stations was expressed by the Jaccard index. Forty-three species were recorded, 21 of these being present in the lotic stretch, 21 in the transition stretch and 30 in the lentic stretch. Salvinia auriculata Aubl. and Paspalum repens P.J. Bergius occurred in $100 \%$ of the sampling units. The lotic and transition regions were predominantly colonized by emergent and amphibious macrophytes, whereas the lentic region was colonized by plants with floating and submersed habits. The analysis showed a high similarity $(>50 \%)$ between lotic and transition stretches, and low similarity $(<25 \%)$ between these and the lentic stretch.

Key-words: floristics, habit, reservoirs, similarity.
\end{abstract}

\section{Introdução}

Os ecossistemas aquáticos continentais são locais importantes para a conservação biológica, pois $9,5 \%$ da riqueza de espécies existentes na Terra colonizam esses ambientes (Balian et al. 2008). Em meio à diversidade de ecossistemas aquáticos encontrados no Brasil, os reservatórios são os que apresentam maior importância social, pois viabilizam o progresso material da população humana e a sustentabilidade dos recursos hídricos (Esteves 1998; Pegorini et al. 2005). Esses mananciais tornam

\footnotetext{
${ }^{1}$ Universidade Federal Rural de Pernambuco, Depto. Biologia, R. Dom Manoel de Medeiros s/n, Dois Irmãos, 52171-900, Recife, PE.

${ }^{2}$ Universidade Federal Rural de Pernambuco, Depto. Pesca e Aquicultura, R. Dom Manoel de Medeiros s/n, Dois Irmãos, 52171-900, Recife, PE.

${ }^{3}$ Universidade Federal do Piauí, Depto. Biologia, R. Cícero Eduardo s/n, Junco, 64600-000, Picos, PI.

${ }^{4}$ Organização Pan-americana de Saúde (OPAS)/Fundação Nacional de Saúde (FUNASA), Av. Conselheiro Rosa e Silva 1489, 52050-020, Recife, PE.

${ }^{5}$ Autor para correspondência: jrbio10@hotmail.com
} 
o potencial hidroelétrico dos rios aproveitável, possibilitam seu uso como vias navegáveis e viabilizam o abastecimento público e industrial (Júlio-Jr. et al. 2005; Tundisi 2007).

Além de seu valor social, os reservatórios desempenham papéis estruturais e funcionais no ecossistema, pois contemplam uma grande diversidade de ambientes e organismos aquáticos (Thomaz et al. 1999; Agostinho et al. 2005; Bini et al. 2005; Tundisi \& Tundisi 2008). Dentre as espécies que colonizam os reservatórios estão às plantas aquáticas, as quais são importantes para a produção de matéria orgânica e ciclagem de nutrientes no ambiente, servem de substrato para o perifiton e propiciam abrigo e proteção a outros organismos aquáticos e/ou anfíbios (Esteves 1998; Pott \& Pott 2000; Pompêo \& Moschini-Carlos 2003; Thomaz \& Cunha 2010).

Ainda que a importância das plantas aquáticas para a ecologia dos reservatórios do Nordeste do Brasil tenha sido bem enfatizada na literatura (França et al. 2003; Moura-Júnior et al. 2009, 2010; Henry-Silva et al. 2010), o entendimento dos padrões e processos relacionados à biodiversidade desses ecossistemas ainda representa um desafio à ecologia teórica e aplicada. Essencialmente, os reservatórios são caracterizados por apresentarem um contínuo fluxo de água no sentido nascente-barragem que propicia a discriminação de três zonas ou regiões (lótica, de transição e lêntica), com distintas estruturas físicas, químicas e biológicas (Thornton 1990; Pompêo 1999; Mormul et al. 2010).

A região lótica dos reservatórios é caracterizada por apresentar água com transparência reduzida, elevada turbidez e baixa concentração de oxigênio dissolvido, enquanto que a região lêntica apresenta águas transparentes, baixa turbidez e níveis elevados de oxigênio dissolvido, sendo a região de transição um ecótono entre os trechos lótico e lêntico (Thornton 1990; Costa 2004). Esse gradiente rio-barragem tem promovido alterações significativas nos padrões da biodiversidade de plantas aquáticas de reservatórios do Sudeste do Brasil (Thomaz et al. 2003; Pompêo 2008), o que sugere respostas similares para reservatórios de outras regiões tropicas, como o semi-árido nordestino.

Embora se reconheça que as plantas aquáticas possam constituir guildas ao longo do gradiente riobarragem dos reservatórios, não existe um consenso acerca dos fatores que determinam os padrões de riqueza, composição florística e formas biológicas desses vegetais. Alguns pesquisadores acreditam que a especificidade das condições abióticas da água (Murphy et al. 2003; Thomaz et al. 2003; Ferreira et al. 2010; Sousa et al. 2010) ou o regime de chuvas (Neiff 1975, 1978; Pott \& Pott 2003) podem interferir nos padrões bióticos das plantas aquáticas. Contudo, outros ecólogos entendem que a formação das guildas de macrófitas aquáticas pode estar relacionada às características biogeográficas (Ferreira et al. 2011) ou aos processos eco-fisiológicos das espécies (HenrySilva \& Camargo 2005; Silva \& Zickel 2010).

Assim, o conhecimento da composição florística, riqueza e formas biológicas de plantas aquáticas ao longo do eixo rio-barragem de um reservatório é capaz de fornecer informações importantes acerca do comportamento das espécies nesse tipo de ecossistema, e com isso ampliar o conhecimento sobre os padrões da biodiversidade desses vegetais em reservatórios tropicais. Nesse contexto, o presente estudo teve por objetivos: (I) determinar a variação na riqueza de plantas aquáticas entre as diferentes regiões (lótica, de transição e lêntica) de um reservatório do semi-árido Nordestino; (II) avaliar a distribuição das formas biológicas ao longo do eixo rio-barragem do reservatório; (III) analisar se ocorre ou não similaridade florística entre essas regiões; (IV) e identificar quais são as espécies mais frequentes ao longo do eixo rio-barragem do reservatório.

\section{Material e Métodos}

O reservatório da Usina Hidroelétrica (UHE)

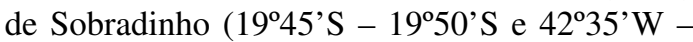
$42^{\circ} 40^{\prime} \mathrm{W}$ ) situa-se nos trechos médio e submédio do rio São Francisco, entre os municípios de Barra e Juazeiro, ambos localizados na Bahia (Fig. 1). O lago artificial de Sobradinho é um dos maiores reservatórios hidrelétricos do mundo, com uma superfície de espelho d'água de $4.214 \mathrm{~km}^{2}$ (Godinho \& Godinho 2003) que pode ser reduzida em até $70 \%$ no período de estiagem, e uma capacidade de armazenamento de 30,1 bilhões de metros cúbico de água. O regime anual de chuvas no reservatório de Sobradinho é distribuído irregularmente entre os meses de outubro/novembro e maio/junho, com pico em março e janeiro, sendo o período de estiagem compreendido entre junho e setembro (Costa 2004). A pluviosidade média no período chuvoso varia de $20 \mathrm{~mm} / \mathrm{km}^{2}$ (setembro/outubro e maio/junho) a mais de $450 \mathrm{~mm} / \mathrm{km}^{2}$ (março e janeiro), enquanto no período de estiagem não ultrapassa os $20 \mathrm{~mm} / \mathrm{km}^{2}$ (Costa 2004). 
As amostragens florísticas foram realizadas durante os bimestres de junho-julho de 2008 e janeiro/ fevereiro de 2009, a fim de abranger os períodos de chuva e estiagem, respectivamente. Foram estabelecidas seis estações de amostragem ao longo do eixo riobarragem do reservatório: estações 1 (1051'20,32”'S / $\left.42^{\circ} 55^{\prime} 45,14^{\prime \prime} \mathrm{W}\right)$ e $2\left(10^{\circ} 44^{\prime} 37,71^{\prime \prime} \mathrm{S} / 042^{\circ} 43^{\prime} 3,67^{\prime}\right.$ 'W) na região lótica; estações 3 ( $10^{\circ} 2$ '23,93”S /

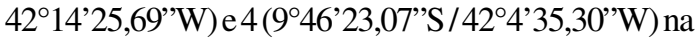
região de transição; e estações $5\left(9^{\circ} 18^{\prime} 36,83^{\prime \prime} \mathrm{S} /\right.$ 40 58'26,38’W) e 6 (9'12'51,09”'S / 40 58'58,03”'W) na região lêntica (Fig.1). Para cada estação, foram plotadas duas parcelas amostrais, uma na margem esquerda e outra na direita, ambas de $20 \times 40 \mathrm{~m}$ (no sentido margem-espelho d'água), estando o ponto inicial localizado a $0,5 \mathrm{~m}$ da margem.

Foram coletados ramos ou indivíduos (em estágio fértil ou vegetativo) das espécies presentes nas parcelas, através de amostragem expedita (com duração média de 45 min por parcela, em cada expedição de coleta), segundo a metodologia de Fidalgo \& Bononi (1984), sem delineamento amostral.

A lista das espécies seguiu a proposta de classificação das famílias reconhecidas pelo APG II (2003) para as Angiospermas, por Smith et al. (2006) para as Pteridófitas, e Buck \& Goffinet (2000) para as Briófitas. A identificação das espécies foi fundamentada em bibliografia especializada e na compilação com os acervos dos herbários Professor Vasconcelos Sobrinho (PEUFR/UFRPE) e da Empresa Pernambucana de Pesquisa Agropecuária (IPA), ambos localizados em Recife - PE. Para a classificação das formas biológicas das macrófitas coletadas, utilizou-se a proposta de Irgang et al. (1984). Os espécimes coletados foram incorporados ao acervo biológico particular da CHESF, o qual se encontra disponível na sede da Instituição, em Recife.

Os dados de riqueza específica e composição florística de cada região do reservatório nos diferentes períodos sazonais foram obtidos através do somatório dos resultados encontrados nas parcelas de cada região, nos bimestres analisados. Quanto à análise dos dados, calculou-se o coeficiente de variação para avaliar se ocorreu diferença no número de espécies entre as diferentes regiões do reservatório, a partir da média e do desvio padrão da riqueza entre as regiões, em cada período sazonal. Em seguida, ordenaram-se os dados florístico através da Análise de Correspondência Destendenciada (DCA) para verificar as diferenças entre as regiões do reservatório e quais as formas

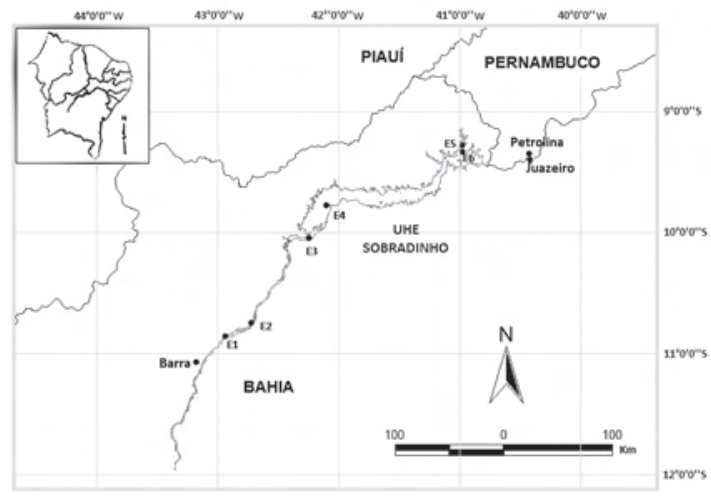

Figura 1 - Mapa da área de abrangência do reservatório de Sobradinho com a localização das 06 estações de coleta. Figure 1 - Map of the area under influence of Sobradinho Reservoir, with location of 06 stations.

biológicas estiveram mais associadas a cada região, utilizando-se o programa CANOCO versão 4.5 (Ter Braak \& Smilauer 2002). Para a melhor visualização dos perfis obtidos, através da DCA, foram retiradas do gráfico as espécies menos correlacionadas com os eixos da ordenação. A avaliação da similaridade florística entre as regiões do reservatório e entre os períodos sazonais foi realizada através de uma análise de agrupamento. A matriz de composição florística foi submetida à análise de similaridade pelo índice de Jaccard (Magurran 2004) e ordenada pelo método WPGMA, através do software PRIMER 6.0 (Clarke \& Gorley 2006). Para testar a consistência dos agrupamentos foi utilizado o método de permutação Monte Carlo com 2000 replicações e $\alpha$ $=1 \%$, através do programa RandMat versão 1.0 (Manly 1997). Com base no registro de campo foram calculados os percentuais de ocorrência das espécies ao longo do reservatório de Sobradinho, segundo a metodologia proposta por Mateucci \& Colma (1982).

\section{Resultados}

Foram inventariadas 43 espécies, distribuídas em 32 gêneros e 25 famílias (Tab. 1). As famílias Cyperaceae e Convolvulaceae foram as mais representativas na riqueza das regiões lótica e de transição do reservatório, contemplando juntas nove espécies para essas regiões. Já para a estação lêntica, a família Salviniaceae foi a que apresentou um maior número de espécies (quatro), correspondendo a $9,30 \%$ do total de macrófitas aquáticas identificadas nas áreas estudadas (Fig. 2). 
Tabela 1 - Lista de espécies (por família), forma biológica (FB) e percentual de freqüência de ocorrência (\%) das espécies de macrófitas aquáticas identificadas nas diferentes regiões do reservatório de Sobradinho - BA, nos meses de chuva e estiagem. (+) presença; (-) ausência. AN - anfíbia; EM - emergente; FL - flutuante livre; FF - flutuante fixa; SL - submersa livre; SF - submersa fixa; EP - Epífita.

Table 1 - List of aquatic macrophytes species (by family), biological form (FB) and percent occurrence frequency, identified in the different stretches of Sobradinho Reservoir - BA, in rainy and drought months. (+) presence; (-) absence. AN - amphibian; EM - emergent, FL - free-floating, FF - rooted-floating, SL - free-submersed; SF - rooted-submersed; EP - epiphytes.

\begin{tabular}{|c|c|c|c|c|c|c|c|c|c|}
\hline Táxon & \multicolumn{2}{|c|}{ Lótico } & \multicolumn{2}{|c|}{ Transição } & \multicolumn{2}{|c|}{ Lêntico } & \multirow[t]{2}{*}{ FB } & $\%$ & \multirow[t]{2}{*}{ Número de Coletor } \\
\hline & Estiagem & Chuvoso & Estiagem & Chuvoso & Estiagem & Chuvoso & & & \\
\hline
\end{tabular}

\section{ALISMATACEAE}

Echinodorus tenellus Buchenau

\section{APOCYNACEAE}

Roulinia montevidensis (Spreng.) Malme.

\section{ARACEAE}

Pistia stratiotes L.

Wolffia brasiliensis Wedd.

\section{BRASSICACEAE}

Cleome spinosa Jacq

\section{CABOMBACEAE}

Cabomba aquatica Aubl.

\section{CONVOLVULACEAE}

Ipomoea asarifolia Roem. \& Schult.

\section{Ipomoea batatoides Choisy}

Ipomoea carnea Jacq.

Ipomoea subrevoluta Choisy

CUCURBITACEAE

Cucumis sp.

\section{CYPERACEAE}

Cyperus odoratus $\mathrm{L}$.

\begin{tabular}{|c|c|c|c|c|c|c|c|c|}
\hline - & - & - & - & - & + & EM1 & 16,67 & Moura Jr., E.G. 038 \\
\hline - & - & - & - & + & - & EP1 & 16,67 & Moura Jr., E.G. 056 \\
\hline- & - & - & - & - & + & FL1 & 16,67 & Abreu, M.C. 020 \\
\hline- & - & - & - & + & - & FL2 & 16,67 & Moura Jr., E.G. 014 \\
\hline+ & - & + & - & - & - & AN1 & 33,33 & Moura Jr., E.G. 060 \\
\hline- & - & - & - & - & + & $\mathrm{SF} 1$ & 16,67 & Moura Jr., E.G. 079 \\
\hline - & + & - & + & - & - & EM2 & 33,33 & Moura Jr., E.G. 050 \\
\hline+ & - & + & + & - & - & AN2 & 50,00 & Moura Jr., E.G. 048 \\
\hline- & + & - & + & + & + & AN3 & 66,67 & Moura Jr., E.G. 043 \\
\hline+ & - & + & - & - & - & EM3 & 33,33 & Moura Jr., E.G. 025 \\
\hline- & - & - & - & + & + & $\mathrm{EP} 2$ & 33,33 & Moura Jr., E.G. 007 \\
\hline+ & - & + & - & + & - & EM4 & 50,00 & Moura Jr., E.G. 044 \\
\hline
\end{tabular}




\begin{tabular}{|c|c|c|c|c|c|c|c|c|c|}
\hline \multirow[t]{2}{*}{ Táxon } & \multicolumn{2}{|c|}{ Lótico } & \multicolumn{2}{|c|}{ Transição } & \multicolumn{2}{|c|}{ Lêntico } & \multirow[t]{2}{*}{ FB } & \multirow[t]{2}{*}{$\%$} & \multirow[t]{2}{*}{ Número de Coletor } \\
\hline & Estiagem & Chuvoso & Estiagem & Chuvoso & Estiagem & Chuvoso & & & \\
\hline Cyperus sp. & + & - & - & - & - & - & EM5 & 16,67 & Moura Jr., E.G. 049 \\
\hline Eleocharis minima Kunth & + & - & + & - & - & - & EM7 & 33,33 & Moura Jr., E.G. 022 \\
\hline Eleocharis sp. & + & - & + & - & - & - & EM8 & 33,33 & Moura Jr., E.G. 051 \\
\hline Oxycaryum cubense (Poepp. \& Kunth) Palla & + & - & - & - & + & + & EM9 & 50,00 & Moura Jr., E.G. 002 \\
\hline Croton rhamnifolius Kunth & + & - & - & + & - & - & AN4 & 33,33 & Abreu, M.C. 031 \\
\hline \multicolumn{10}{|l|}{ HYDROCHARITACEAE } \\
\hline Egeria densa Planchon & - & + & + & - & - & + & $\mathrm{SF} 2$ & 50,00 & Abreu, M.C. 025 \\
\hline Valisneria sp. & + & - & - & - & - & - & $\mathrm{SF} 3$ & 16,67 & Moura Jr., E.G. 035 \\
\hline \multicolumn{7}{|l|}{ HYDROLEACEAE } & AN5 & 33,33 & Abreu, M.C. 032 \\
\hline \multicolumn{10}{|l|}{ LENTIBULARIACEAE } \\
\hline $\begin{array}{l}\text { LIMNOCHARITACEAE } \\
\text { Hydrocleys nymphoides (Willd.) Buchenau }\end{array}$ & - & - & - & - & + & - & EM10 & 16,67 & Abreu, M.C. 036 \\
\hline $\begin{array}{l}\text { MALVACEAE } \\
\text { Melochia pyramidata } \mathrm{L} .\end{array}$ & - & - & + & - & - & - & AN6 & 16,67 & Abreu, M.C. 002 \\
\hline $\begin{array}{l}\text { NYMPHAEACEAE } \\
\text { Nymphaea amazonum Mart. \& Zucc. } \\
\text { ONAGRACEAE }\end{array}$ & - & - & - & - & + & - & $\mathrm{FF} 1$ & 16,67 & Abreu, M.C. 038 \\
\hline Ludwigia helminthorrhiza (Mart.) H. Hara & - & - & - & - & + & + & FL3 & 33,33 & Severi, W. 006 \\
\hline Ludwigia leptocarpa (Nutt.) H. Hara & - & - & + & - & + & + & EM11 & 50,00 & Moura Jr., E.G. 047 \\
\hline
\end{tabular}




\begin{tabular}{|c|c|c|c|c|c|c|c|c|c|}
\hline \multirow[t]{2}{*}{ Táxon } & \multicolumn{2}{|c|}{ Lótico } & \multicolumn{2}{|c|}{ Transição } & \multicolumn{2}{|c|}{ Lêntico } & \multirow[t]{2}{*}{ FB } & \multirow[t]{2}{*}{$\%$} & \multirow[t]{2}{*}{ Número de Coletor } \\
\hline & Estiagem & Chuvoso & Estiagem & Chuvoso & Estiagem & Chuvoso & & & \\
\hline \multicolumn{10}{|l|}{ PLANTAGINACEAE } \\
\hline Bacopa aquatica Aubl. & - & - & - & - & + & + & EM12 & 33,33 & Moura Jr., E.G. 027 \\
\hline \multicolumn{10}{|l|}{ POACEAE } \\
\hline Hymenachne amplexicaulis (Rudge) Nees & + & - & + & - & - & - & AN7 & 33,33 & Moura Jr., E.G. 045 \\
\hline Paspalum repens P.J. Bergius & + & + & - & + & + & + & EM13 & 83,33 & Moura Jr., E.G. 003 \\
\hline \multicolumn{10}{|l|}{ POLYGONACEAE } \\
\hline Polygonum ferrugineum Wedd & + & - & + & + & + & - & EM14 & 66,67 & Abreu, M.C. 028 \\
\hline \multicolumn{10}{|l|}{ PONTEDERIACEAE } \\
\hline Eichhornia azurea Kunth & + & + & + & + & - & + & FL4 & 83,33 & Abreu, M.C. 019 \\
\hline Eichhornia crassipes (Mart.) Solms & + & + & - & + & + & + & FL5 & 83,33 & Severi, W. 007 \\
\hline \multicolumn{10}{|l|}{ PTERIDACEAE } \\
\hline Ceratopteris pteridoides (Hook.) Hieron. & - & - & - & - & + & - & EM15 & 16,67 & Moura Jr., E.G. 032 \\
\hline \multicolumn{10}{|l|}{ RICCIACEAE } \\
\hline Ricciocarpus natans (L.) Corda & - & - & + & - & + & + & FL6 & 50,00 & Moura Jr., E.G. 009 \\
\hline \multicolumn{10}{|l|}{ SALVINIACEAE } \\
\hline Azolla caroliniana Willd. & - & - & - & - & + & - & FL7 & 16,67 & Severi, W. 001 \\
\hline Azolla filiculoides Lam. & - & - & - & - & + & - & FL8 & 16,67 & Moura Jr., E.G. 057 \\
\hline Salvinia auriculata Aubl. & + & + & + & + & + & + & FL9 & 100,00 & Abreu, M.C. 006 \\
\hline Salvinia minima Baker & - & - & - & - & + & - & FL10 & 33,33 & Moura Jr., E.G. 004 \\
\hline Salvinia oblongifolia Martius & + & - & + & - & - & - & FL11 & 33,33 & Moura Jr., E.G. 054 \\
\hline \multicolumn{10}{|l|}{ THELYPTERIDACEAE } \\
\hline Thelypteris interrupta (Willd.) K. Iwats. & - & - & - & - & + & + & EM16 & 33,33 & Abreu, M.C. 040 \\
\hline \multicolumn{10}{|l|}{ TYPHACEAE } \\
\hline Typha domingensis Pers. & - & - & - & - & + & + & EM17 & 33,33 & Abreu, M.C. 021 \\
\hline
\end{tabular}




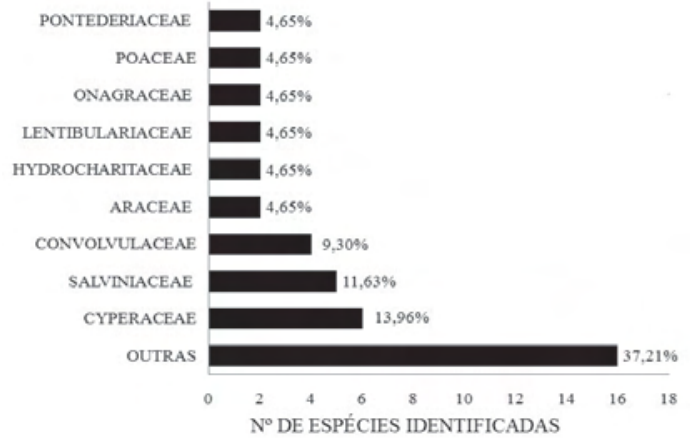

Figura 2 - Riqueza e distribuição percentual das famílias de macrófitas aquáticas mais representativas, no reservatório de Sobradinho - BA.

Figure 2 - Richness and percent distribution of most representative aquatic macrophytes in Sobradinho Reservoir - BA.

Dentre as espécies de macrófitas aquáticas identificadas no reservatório de Sobradinho $22 \mathrm{spp}$. $(51,16 \%)$ foram observadas exclusivamente no período de estiagem e seis $(13,95 \%)$ no chuvoso, sendo a riqueza específica total para os períodos de estiagem e chuvoso de 39 e 22 spp., respectivamente. No período de estiagem, 18 táxons ocorreram na região lótica, 16 na de transição e 25 na lêntica, enquanto que, no período chuvoso foram registradas sete espécies na região lótica, nove na de transição e 18 na lêntica. A análise do coeficiente de variação $(\mathrm{CV})$ confirmou as diferenças sazonais quanto à riqueza de macrófitas aquáticas das três regiões do reservatório, com uma diferença mais acentuada entre as regiões lêntica-lótica e transição-lêntica, no período chuvoso (Tab. 2).

Quanto à distribuição das formas biológicas, observou-se que as regiões lótica e de transição contemplaram, predominantemente, espécies anfíbias e emergentes, diferindo da região lêntica, onde espécies flutuantes, emergentes e submersas foram mais evidentes (Fig. 3). Com uma explicabilidade em $51,1 \%$ nos dois primeiros eixos, a Análise de Correspondência Destendenciada (DCA) confirmou esse padrão. Foram estabelecidos dois grupos em função da ocorrência ou não das espécies. O grupo (A) foi constituído pelas regiões lótica e de transição, as quais convergiram em função das espécies anfíbias (AN) e emergentes (EM). O grupo (B) esteve representado pela região lêntica, a qual foi colonizada por uma maior diversidade de formas biológicas (Fig. 4).
Tabela 2 - Média, desvio padrão e coeficiente de variação $(\mathrm{CV})$ da riqueza de macrófitas aquáticas nas diferentes regiões do reservatório de Sobradinho $-\mathrm{BA}$. $\mathrm{PE}=$ período de estiagem; $\mathrm{PC}=$ período chuvoso.

Table 2 - Average, standard deviation, variation coefficient (CV) of aquatic macrophytes richness in the different stretches of Sobradinho Reservoir - BA. PE = dry season; $\mathrm{PC}=$ rainy season.

\begin{tabular}{lcccccc}
\hline Regiões & Média & \multicolumn{3}{c}{$\begin{array}{c}\text { Desvio } \\
\text { padrão }\end{array}$} & \multicolumn{2}{c}{ CV } \\
\hline & PE & PC & PE & PC & PE & PC \\
\hline Lotico-Transição & 17,0 & 8,0 & 1,4 & 1,4 & $8,31 \%$ & $17,68 \%$ \\
Lotico-Lêntico & 21,5 & 12,5 & 4,9 & 7,8 & $23,02 \%$ & $62,23 \%$ \\
Lêntico-Transição & 20,5 & 13,5 & 6,4 & 6,4 & 31,04 & $47,14 \%$ \\
\hline
\end{tabular}

De acordo com o teste de Monte Carlo, as comunidades de macrófitas que apresentaram índices de Jaccard acima de $44 \%$ foram consideradas significativamente similares, quanto à estrutura florística. Nesse sentido, a variação da composição florística de plantas aquáticas entre as regiões do reservatório de Sobradinho foi comprovada pelos índices de similaridade que evidenciaram alta similaridade (> 45\%) entre os ecossistemas lótico e de transição, e baixa similaridade $(<25 \%)$ entre esses ambientes e a região lêntica (Fig. 5), sendo esses padrões observados para os diferentes períodos sazonais.

Quanto à frequência de ocorrência, constatou-se que apenas oito espécies ocorreram nas três regiões do reservatório: Salvinia auriculata; Paspalum repens; Eichhornia azurea; Eichhornia crassipes; Polygonum ferrugineum; Ipomoea carnea; Cyperus odoratus; e Egeria densa, sendo todas reconhecidas como espécies daninhas em ecossistemas aquáticos continentais. Desse total apenas duas espécies (Salvinia auriculata, Paspalum repens) apresentaram $100 \%$ de presença entre as estações amostrais. Por outro lado, 13 espécies (30,23\%) apresentaram apenas um registro de ocorrência no reservatório de Sobradinho, sendo que desse total, 11 ocorreram na região lêntica (oito no período de estiagem e três no chuvoso), duas na lótica e uma na de transição (todas no período de estiagem) (Tab. 1). 


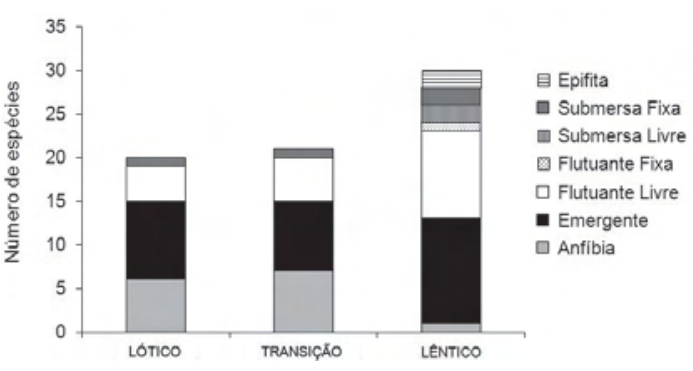

Figura 3 - Distribuição numérica das macrófitas aquáticas do reservatório de Sobradinho - BA, pelas diferentes categorias de formas biológicas.

Figure 3 - Numeric distribution of aquatic macrophytes of Sobradinho Reservoir - BA by different biological form categories

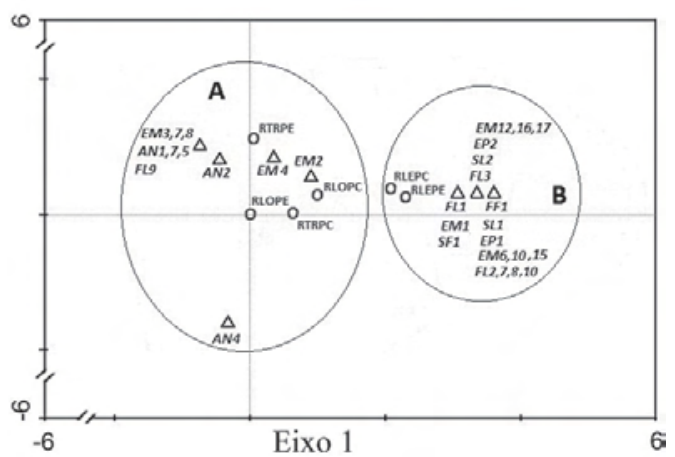

Figura 4 - Representação da Análise de Correspondência Destendenciada - DCA (com explicabilidade de $51,1 \%$ para os dois primeiros eixos), segundo a distribuição das formas biológicas em cada região do reservatório de Sobradinho - BA. RLOPS - região lótica, período seco; RLOPC - região lótica, período chuvoso; RTRPS - região de transição, período seco; RTRPC - região de transição, período chuvoso; RLEPS - região lêntica, período seco; RLEPC - região lêntica, período chuvoso; AN anfíbia; EM - emergente; FL - flutuante livre; FF flutuante fixa; SL - submersa livre; SF - submersa fixa; EP - epífita.

Figure 4 - Detrended Correspondence Analysis - DCA (as explained $51.1 \%$ for the first two axes) according biological form distribution of each stretch of Sobradinho Reservoir BA. RLOPS - lotic stretch, drought season; RLOPC - lotic stretch, rainy season; RTRPS - transition stretch, drought season; RTRPC - transition stretch, rainy season; RLEPS lentic stretch, rainy season; RLEPC - lentic stretch, rainy season; AN - amphibian; EM - emergent, FL - free-floating, $\mathrm{FF}$ - rooted-floating, SL - free-submersed; SF - rootedsubmersed; EP - epiphytes.

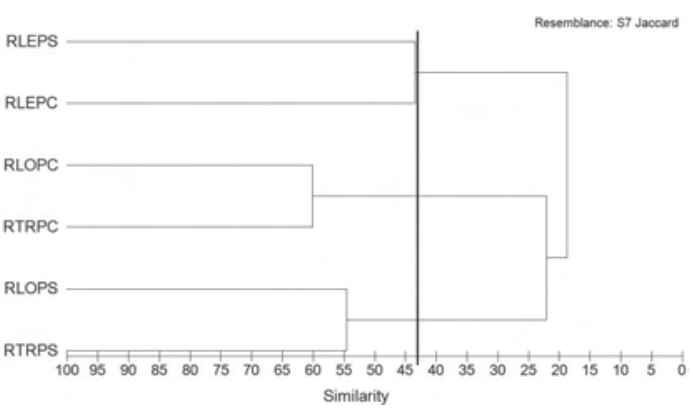

Figura 5 - Dendrograma da similaridade florística das macrófitas aquáticas, entre as regiões do reservatório de Sobradinho-BA, acompanhado do teste de permutaçãoMonte Carlo $=0,25$ (com 2.000 replicações, $\alpha=1 \%)$. RLOPS região lótica, período seco; RLOPC - região lótica, período chuvoso; RTRPS - região de transição, período seco; RTRPC -região de transição, período chuvoso; RLEPS-região lêntica, período seco; RLEPC - região lêntica, período chuvoso.

Figure 5 - Dendrogram of floristic similarity of aquatic macrophytes along the Sobradinho Reservoir - BA, by Monte Carlo permutation test $=0.25$ (with 2,000 replications, $\alpha=$ $1 \%)$. RLOPS - lotic stretch, drought season; RLOPC - lotic stretch, rainy season; RTRPS - transition stretch, drought season; RTRPC - transition stretch, rainy season; RLEPS - lentic stretch, rainy season; RLEPC - lentic stretch, rainy season.

\section{Discussão}

O presente estudo apresentou uma riqueza de macrófitas aquáticas semelhante àquela constatada em dois reservatórios do Parque Estadual de Dois Irmãos - PE (Moura-Júnior et al. 2009), onde foram registradas 48 espécies, bem como a da bacia do rio Apodi/Mossoró (HenrySilva et al. 2010), na qual 40 táxons foram inventariados. No entanto, o número de espécies observadas em Sobradinho foi relativamente baixo em comparação com o estudo realizado em seis reservatórios do semi-árido baiano (França et al. 2003), com registro de 121 espécies. Segundo Silva \& Zickel (2010), a região do semi-árido nordestino possui uma grande diversidade de reservatórios com distintos aspectos hidrológicos, e aqueles que apresentam características perenes, com baixos níveis tróficos que sofrem pequena influencia de variações hidrológicas (semelhante aos analisados por França et al. 2003), abrigam uma flora aquática vascular diversificada.

Cyperaceae também foi evidenciada como a família com maior riqueza específica em ecossistemas aquáticos lóticos da Bahia (França et 
al. 2003), do Rio Grande do Norte (Henry-Silva et al. 2010) e de Pernambuco (Moura-Júnior et al. 2009). Atualmente, estima-se que existam cerca de 5.000 espécies de Cypereaceae (Govaerts $e t$ al. 2007) distribuídas em uma grande variedade de ambientes aquáticos e áreas de ecótono (Sobral-Leite et al. 2010; Henry-Silva et al. 2010). Goetghebeur (1998) afirma que a elevada representatividade florística desta família está associada à eficiência na propagação vegetativa dos seus representantes, os quais apresentam um sistema subterrâneo formado por rizomas, tubérculos ou estolões, o que foi comum para as espécies de Cyperaceae registradas no reservatório de Sobradinho.

Assim como observado no presente estudo, a família Salviniaceae esteve entre as mais representativas na flora aquática da lagoa Silvana - MG (Pivari et al. 2008) e em lagoas do Pantanal (Pott \& Pott 2003). Segundo Moran (1995), a preferência das Salviniaceae por ambientes lênticos (e.g. lagos e lagoas) ocorre em função da estratégia reprodutiva (por esporos) e da forma biológica (flutuante livre) adotada pelos seus representantes.

Ao inventariar as macrófitas aquáticas de uma lagoa do semiárido baiano, Neves et al. (2006) observaram um maior número de espécies no período chuvoso, não corroborando com os dados de riqueza do presente estudo. Sabe-se que a chuva, e, consequentemente, a alteração do regime hidrológico representam forças seletivas capazes de promover significativas flutuações na estrutura da macroflora aquática (Santos \& Thomaz 2007; Thomaz et al. 2009; Sousa et al. 2010). Entretanto, os processos de colonização, sucessão e estabilidade das comunidades de plantas aquáticas também podem ser influenciados pelas características abióticas da água, como por exemplo, pela disponibilidade de luz, pH, condutividade elétrica e concentração de nutrientes fosfatados e nitrogenados (Murphy et al. 2003). De acordo com Esteves (1998) a riqueza das macrófitas aquáticas está inversamente relacionada, dentre outros fatores, com o estado trófico da água. Levando em consideração que o reservatório de Sobradinho apresenta um baixo índice trófico durante o período de estiagem, quando comparado ao chuvoso (Costa 2004), a maior riqueza florística registrada no presente estudo, durante o primeiro período, pode estar relacionada às características abióticas da água.

Moura-Júnior et al. (2010) constataram diferenças quanto ao padrão de riqueza, composição florística e forma biológica da flora aquática vascular de ambientes lênticos e lóticos do trecho sub-médio rio São Francisco, os quais convergiram com com os dados relacionados no presente estudo. Segundo esses pesquisadores, a correnteza foi capaz de carrear os baceiros de macrófitas aquáticas flutuantes de ambientes lóticos para lênticos, tendo algumas das características hidrológicas dos ambientes lênticos (e.g. menor turbidez e maior transparência) favorecido a colonização de uma flora aquática rica e heterogênea.

A ocorrência das espécies $S$. auriculata, E. crassipes, E. azurea, $P$. repens, $P$. ferrugineum, I. carnea, $C$ odoratus e E. densa ao longo de todo o gradiente rio-barragem do reservatório de Sobradinho pode ser considerado um evento comum. De acordo com Pott \& Pott (2000), essas espécies apresentam ampla distribuição e/ou são oportunistas em diversos ambientes aquáticos continentais. É importante ressaltar que, sob determinadas condições, o crescimento excessivo da vegetação aquática pode se tornar um problema para determinados usos da água, como irrigação, abastecimento de cidades e indústrias, navegação e geração de energia (Marcondes et al. 2003; Pereira et al. 2008). Nesse sentido, a elevada presença das referidas espécies pode sinalizar para futuros problemas operacionais na UHE Sobradinho ( $e$. $g$. interrupção na geração de energia), caso não sejam desenvolvimentos estudos continuados sobre a produtividade primária dessas espécies. Entretanto, Moura-Júnior et al. (2010), ao estudarem a biologia das macrófitas aquáticas da região do médio São Francisco, não registraram estandes em tamanho e biomassa que pudessem corroborar com essa problemática.

Em síntese, a amostragem nas três regiões do reservatório de Sobradinho evidenciou diferenças quanto à riqueza, composição florística e formas biológicas das plantas aquáticas, sendo as regiões lótica e de transição mais similares, quando comparadas com a região lêntica. Ainda que o reservatório de Sobradinho apresente registros de macrófitas aquáticas daninhas, esse 
contempla uma grande biodiversidade de plantas aquáticas, e nesse sentido, pode ser considerado essencial para a manutenção de parte da biota aquática do trecho sub-médio do rio São Francisco, além de apresentar grande relevância social e ecológica para a região Nordeste do Brasil. Os resultados obtidos no presente estudo sugerem ainda que cada região do reservatório de Sobradinho é constituída por uma comunidade de plantas aquáticas distinta, sendo a especificidade das condições abióticas da água (turbidez, transparência, tempo de residência e concentração de oxigênio dissolvido) o principal fator estruturador de cada comunidade.

\section{Agradecimentos}

Os autores agradecem à Fundação Apolônio Salles de Desenvolvimento Educacional (FADURPE) em parceria com a Companhia Hidro Elétrica do São Francisco (CHESF), pelo apoio financeiro e logístico concedidos para a realização dessa pesquisa.

\section{Referências}

Agostinho, A.A.; Thomaz, S.M. \& Gomes, L.C. 2005. Conservation of the biodiversity of Brazil's inland waters. Conservation Biology 19: 646-652.

APG II - Angiosperm Phylogeny Group. 2003. An update of the angiosperm phylogeny group classification of the orders and families of flowering plants: APG II. Botanical Journal of the Linnean Society 141: 399-436.

Balian, E.V.; Segers, H.; Lévèque, C. \& Martens, K. 2008. The freshwater animal diversity assessment: an overview of the results. Hydrobiologia 595: 627-637.

Bini, L.M.; Oliveira, L.G.; Souza, D.C.; Carvalho, P \& Pinto, M.P. 2005. Patterns of the aquatic macrophyte cover in Cachoeira Dourada Reservoir (GO-MG). Brazilian Journal of Biology 65: 19-24.

Buck, W.R. \& Goffinet, B. 2000. Morphology and classification of mosses. In: Shaw, J.A. \& Goffinet, B. (eds.). Bryophyte Biology. Cambridge University Press, Cambridge. Pp. 72-124.

Clarke, K.R.; Gorley, R.N. 2006. PRIMER Plymouth routines in multivariate ecological research. Ver. 6: User Manual/Tutorial. PRIMER-E, Plymout. 190p.

Costa, B.D.F. 2004. Caracterização ambiental e dimensionamento da capacidade de aproveitamento do Reservatório de Sobradinho para a instalação de tanques-rede. Dissertação de Mestrado. Universidade Federal Rural de Pernambuco, Recife. 64p.

Esteves, F.A. 1998. Fundamentos de limnologia. 2ed. Interciência, Rio de Janeiro. 602p.

Ferreira, F.A.; Mormul, R.P.; Pedralli, G.; Pott, V.J.; Pott, A. 2010. Estrutura da comunidade de macrófitas aquáticas em três lagoas do Parque Estadual do Rio Doce, Minas Gerais, Brasil. Hoehnea 37: 43-52.

Ferreira, F.A.; Mormul, R.P.; Thomaz, S.M.; Pott, A. \& Pott, V.J. 2011. Macrophytes in the upper Paraná river floodplain: checklist and comparison with other large South American wetlands. Revista de Biologia Tropical 59: 541-556.

Fidalgo, O. \& Bononi, V.R.L. 1984. Técnicas de coleta, preservação e herborização de material botânico. Instituto de Botânica, São Paulo. 62p.

França, F.; Melo, E.; Neto, A.G.; Araújo, D.; Bezerra, M.; Ramos, H.M.; Castro, I. \& Gomes, D. 2003. Flora vascular de açudes de uma região do semi-árido da Bahia, Brasil. Acta Botanica Brasilica 17: 549-559.

Godinho, A.L. \& Godinho, H.P. 2003. Breve visão do São Francisco. In: Godinho, H.P. \& Godinho, L.A. (eds.). Águas, peixes e pescadores do São Francisco das Minas Gerais. PUC-Minas, Belo Horizonte. Pp. 15-24.

Goetghebeur, P. 1998. Cyperaceae. In: Kubitzki, K. (ed.). The families and genera of vascular plants. Springer, Berlin. Pp. 141-190.

Govaerts, R.; Simpson, D.; Bruhl, J.; Egorova, T.; Goetghebeur, P. \& Wilson, K. 2007. World checklist of Cyperaceae - Sedges. Royal Botanic Gardens, Kew. 765p.

Henry-Silva, G.G. \& Camargo, A.F.M. 2005. Interações ecológicas entre as macrófitas aquáticas flutuantes Eichhornia crassipes e Pistia stratiotes. Hoehnea 32: 445-452.

Henry-Silva, G.G.; Moura, R.S.T. \& Dantas, L.L.O. 2010. Richness and distribution of aquatic macrophytes in Brazilian semi-arid aquatic ecosystems. Acta Limnologica Brasiliensia 22: 147-156.

Irgang, B.E.; Pedralli, G. \& Waechter, J.I. 1984. Macrófitos aquáticos da Estação Ecológica do Taim, Rio Grande do Sul, Brasil. Roessleria 6: 395-404.

Julio-Jr, H.F.; Thomaz, S.M.; Agostinho, A.A. \& Latini, J.D. 2005. Distribuição e caracterização dos reservatórios. In: Rodrigues, L.; Thomaz, S.M.; Agostinho, A.A. \& Gomes, L.C. Biocenoses em reservatórios: padrões espaciais e temporais. Rima, São Carlos. Pp. 1-16.

Magurran, A.E. 2004. Measuring biological diversity. Blackwell Publishing, Malden. 256p.

Manly, B.F.J. 1997. Randomization, bootstrap and Monte Carlo methods in biology. 2ed. Chapman and Hall, London. 399p.

Marcondes, D.A.S.; Mustafá, A.F. \& Tanaka, R.H. 2003. Estudos para manejo integrado de plantas aquáticas no reservatório de Jupiá. In: Thomaz, M.S. \& Bini, M.L. Ecologia e manejo de macrófitas aquáticas. Eduem, Maringá. Pp. 299-318.

Mateucci, S.D. \& Colma, A. 1982. La Metodologia para el Estudo de la Vegetacion. Collecion de Monografias Cientificas; Série Biologia 22: 1-168. 
Moran, R.C. 1995. Salviniaceae. In: Davidse, G.; Sousa, M. \& Knapp, S. (eds.). Flora Mesoamericana. Vol. 1. Universidad Nacional Autónoma de México, Ciudad de México. Pp. 1-395.

Mormul, R.P.; Ferreira, F.A.; Carvalho, P.; Michelan, T.S.; Silveira, M.J. \& Thomaz, S.M. 2010. Aquatic macrophytes in the large, sub-tropical Itaipu Reservoir. Revista de Biologia Tropical 58: 1437-1452.

Moura-Júnior, E.G; Silva, S.S.L.; Lima, L.F.; Lima, P.B.; Almeida-Jr., E.B.; Pessoa, L.M.; Santos-Filho, F.S.; Medeiros, D.P.W.; Pimentel, R.M.M. \& Zickel, C.S. 2009. Diversidade de plantas aquáticas vasculares em açudes do Parque Estadual de Dois Irmãos (PEDI), Recife-PE. Revista de Geografia 26: 178-293.

Moura-Júnior, E.G.; Abreu, M.C.; Severi, W. \& Lira, G.A.S.T. 2010. Macroflora aquática do Reservatório Sobradinho - BA, trecho sub-médio do Rio São Francisco. In: Moura, A.M.; Araújo, E.L.; Bittencourt-Oliveira, M.C.; Pimentel, R.M.M. \& Albuquerque, U.P. (eds.). Reservatórios do Nordeste do Brasil: biodiversidade, ecologia e manejo. Nuppea, Recife. Pp. 189-212.

Murphy, K.J.; Dickinson, G.; Thomaz, S.M.; Bini, L.M.; Dick, K.; Greaves, K.; Kennedy, M.P.; Livingstone, S.; Mcferran, H.; Milne, J.M; Oldroyd, J. \& Wingfield R.A. 2003. Aquatic plant communities and predictors of diversity in a sub-tropical river floodplain: the upper Rio Paraná, Brazil. Aquatic Botany 77: 257-276.

Neiff, J.J. 1975. Fluctuaciones anuales en la composition fitocenotica y biomassa de la hidrofitia en lagunas islenas del Paraná Medio. Ecosur 2: 153-183.

Neiff, J.J. 1978. Fluctuaciones de la vegetacion acuatica em ambientes del valle de inundacion del Paraná medio. Physis 38: 41-53.

Neves, E.L.; Leite, K.R.B.; França, F. \& Melo, E. 2006. Plantas aquáticas vasculares em uma lagoa de planície costeira no município de Candeias, Bahia, Brasil. Sitientibus, Série Ciências Biológicas 6: 24-29.

Pegorini, E.S.; Carneiro, C. \& Andreoli, C.V. 2005. Mananciais de abastecimento público. In: Andreoli, C.V. \& Carneiro, C. (eds.). Gestão integrada de mananciais de abastecimento eutrofizados. Sanepar/ Finep, Curitiba. Pp. 1-500.

Pereira, S.M.B.; Nascimento, P.R.F.; Sampaio, E.V.B.; Moura-Jr., A.M. \& Oliveira-Carvalho, M.F. 2008. Monitoramento e manejo da macrófita aquática Egeria densa Planchon no nordeste brasileiro Estudo de caso. In: Moura, A.N.; Araújo, E.L. \& Albuquerque, U.P. (eds.). Biodiversidade, potencial econômico e processos eco-fisiológicos em ecossistemas nordestinos. Comunigraf, Recife. Pp. 209-234.

Pivari, M.O.D.; Salimena, F.R.G.; Pott, V.J. \& Pott, A. 2008. Macrófitas aquáticas da Lagoa Silvana, Vale do Rio Doce, Minas Gerais, Brasil. Iheringia 63: 321-327.

Pompêo, M.L.M. 1999. Perspectivas da limnologia no Brasil. Gráfica e Editora União, São Luís. 191p.

Pompêo, M.L.M. \& Moschini-Carlos. 2003. Macrófitas aquáticas e perifíton, aspectos ecológicos e metodológicos. Rima, São Carlos. 130p.

Pompêo, M.L.M. Monitoramento e manejo de macrófitas aquáticas. 2008. Oecologia Brasiliensis 12: 406-424.

Pott, V.J. \& Pott, A. 2000. Plantas aquáticas do Pantanal. Embrapa, Brasília. 404p.

Pott, V.J. \& Pott, A. 2003. Dinâmica da vegetação aquática do Pantanal. In: Thomaz, S.M. \& Bini, L.M. (eds.). Ecologia e manejo de macrófitas aquáticas. Vol. 1. Eduem, Maringá. Pp. 145-162.

Santos, A.M. \& Thomaz, S.M. 2007. Aquatic macrophytes diversity in lagoons of a tropical floodplain: the role of connectivity and water level. Austral Ecology 32: 177-190.

Silva, S.S.L. \& Zickel, C.S. 2010. Macrófitas aquáticas: conceitos e metodologia para os reservatórios nordestinos. In: Moura, A.M.; Araújo, E.L.; Bittencourt-Oliveira, M.C.; Pimentel, R.M.M.; Albuquerque, U.P. (eds.). Reservatórios do Nordeste do Brasil: biodiversidade, ecologia e manejo. Nuppea, Recife. Pp. 71-186.

Smith, A.R.; Pryer, K.M.; Schuettpelz, E.; Korall, P.; Schneider, H. \& Wolf, P.G. 2006. A classification for extant ferns. Taxon 55: 705-731.

Sobral-Leite, M.; Campelo, M.J.A.; Siqueira-Filho, J.A. \& Silva, S.I. 2010. Checklist das macrofitas vasculares de Pernambuco: riqueza de especies, formas biologicas e consideracoes sobre distribuicao. In: Albuquerque, U.P.; Moura, A.N. \& Araujo, E.L. (eds.). Biodiversidade, potencial econômico e processos ecofisiológicos em ecossistemas nordestinos. Vol. 2. Nuppez, Recife. Pp. 253-280.

Sousa, W.T.Z.; Thomaz, S.M. \& Murphy, K.J. 2010. Response of native Egeria najas Planch. and invasive Hydrilla verticillata (L.f.) Royle to altered hydroecological regime in a subtropical river. Aquatic Botany 92: 40-48.

Ter Braak, C.J.F \& Smilauer, P. 2002. CANOCO Reference and CanoDraw for Windows user's guide: Software for canonical community ordination. Microcomputer Power, Ithaca.

Thomaz, S.M.; Bini, L.M.; Souza, M.C.; Kita, K.K. \& Camargo, A.F.M. 1999. Aquatic macrophytes of Itaipu Reservoir, Brazil: survey of species and ecological considerations. Brazilian Archives of Biology and Technology 42: 15-22.

Thomaz, S.M.; Souza, D.C. \& Bini, L.M. 2003. Species richness and beta diversity of aquatic macrophytes in a large subtropical reservoir (Itaipu Reservoir, 
Brazil): the influence of limnology and morphometry. Hydrobiologia 505: 119-128.

Thomaz, S. M.; Carvalho, P.; Padial, A.A. \& Kobayashi, J.T. 2009. Temporal and spatial patterns of aquatic macrophyte diversity in the Upper Paraná River floodplain. Brazilian Journal of Biology 69: 617-625.

Thomaz, S.M. \& Cunha, E.R. 2010. The role of macrophytes in habitat structuring in aquatic ecosystems: methods of measurement, causes and consequences on animal assemblages' composition and biodiversity. Acta Limnologica Brasiliensia 22: 218-236.

Thornton, K.W. 1990. Perspectives on reservoir limnology. In: Thornton, K.W.; Kimmel, B.L. \& Payne, F.E. (eds.). Reservoir limnology: ecological perspectives. Wiley-Interscience, New York. Pp. $1-13$.

Tundisi, J.G. 2007. Exploração do potencial hidrelétrico da Amazonia. Estudos Avançados 21: 109-117.

Tundisi, J.G. \& Tundisi, T.M. 2008. Limnologia. Oficina de Texto, São Paulo. 632p. 